\title{
El ciclo misionero de Hernando de Villafañe (1560-1634) y su relevancia en la Provincia Mexicana de la Compañía de Jesús
}

\author{
Hernando de Villafañe's missionary cicle (1560-1634) and his \\ relevance in the Society of Jesus' Mexican Province
}

Gilberto López Castillo*

Resumen: Hernando de Villafañe (León, Castilla la Vieja 1560 - Sinaloa, 1634), fue uno de los principales actores de la Compañía de Jesús en la época inicial en la misión de Sinaloa (Nueva Vizcaya), por lo que una revisión de la información sobre este personaje sobre el que no existe una biografía actualizada permite contextualizar las distintas facetas de un jesuita cuya fama como misionero ha trascendido, convirtiéndose en referente, no solo del papel de los jesuitas en Sinaloa, sino del desarrollo de la Provincia jesuítica mexicana en momentos fundacionales del trabajo permanente con los indios. Investigar a Villafañe contribuye a un mayor conocimiento de la misión jesuita, su función y organización interna, a la vez que permite comprender los entramados específicos de una institución global.

Palabras clave: Compañía de Jesús, Sinaloa, misión, indios, Provincia Mexicana

\begin{abstract}
Hernando de Villafañe (León, Castilla la Vieja, 1560 - Sinaloa, 1634) was one of the Society of Jesus' main actors in the early years of the order's mission in Sinaloa (New Vizcaya). A review of the information regarding this protagonist, over whom there is no updated biography, allows us to contextualize the differents faces of a Jesuit whose fame as missionary has transcended to our days, becoming a referent not only of the Jesuits in Sinaloa but also of the Mexican Jesuit Province in the founding moments of an enduring missionary work among the Indians. Doing research on Villafañe contributes to enlarge the knowledge of the Jesuit missions, their function and internal organization, and to understand the intricacies of a global institution.
\end{abstract}

\footnotetext{
* Profesor-investigador del Instituto Nacional de Antropología e Historia (INAH-Sinaloa). México. E-mail: gilbertohistory@gmail.com
} 
Key words: Society of Jesus, Sinaloa, Mission, Indians, Mexican Province.

Recibido: 2 de octubre de 2016

Evaluado: 6 de noviembre de 2016 


\section{Introducción}

Si bien el nuestro se trata de un personaje cuya labor más conocida nos lleva a la misión entre los grupos guasaves y en general, de todos los habitantes del bajo río Sinaloa, Hernando de Villafañe tuvo diversos momentos en su participación en la Provincia Mexicana de la Compañía de Jesús que vale la pena destacar, desde los inicios de su formación misionera en la residencia de Pátzcuaro en la provincia de Michoacán hasta uno de los momentos cumbre, como fue el de ser procurador de la Provincia Mexicana en su Congregación General en Roma el año de 1620. La documentación como la historiografía es abundante, de tal forma que su presencia es inmanente en los clásicos de la historiografía jesuítica referida a la provincia Mexicana de la orden, aunque solo excepcionalmente se le estudia de forma central. ${ }^{1}$ Por todo ello, un nuevo balance historiográfico y el rescate de fuentes inéditas nos brindan la posibilidad de ofrecer un punto de vista que busca ser complementario al perfil hoy conocido del misionero peninsular. ${ }^{2}$

Es importante decir que la figura de Villafañe como la de muchos otros misioneros de aquella época que trabajaron por décadas entre los grupos indígenas del noroeste novohispano queda opacada por la significación que para la orden tuvo el martirio del padre Gonzalo de Tapia en 1594, o por el gran interés que la historiografía del siglo XX y aun hoy día ha tenido la figura de Eusebio Francisco Kino, misionero en la pimería alta ${ }^{3}$. Nuestra intención es realizar una caracterización de éste jesuita que contribuya a delinear su perfil como un actor social relevante en la expansión de la monarquía española en el noroeste novohispano.

Cabe decir que gran parte de la información sobre Villafañe se debe en buena medida a los testimonios dispersos en la obra Triunfos de nuestra santa fee, del también misionero jesuita Andrés Pérez de Ribas (Fig. 1 y 2), aunque más particularmente a su nota biográfica "Vida y muerte del muy religioso padre Hernando de Villafañe, que trabajó treinta años en la missión de Cinaloa, donde felizmente acabó su santa vida", que bien puede ser revalorada mediante la complementariedad que ofrecen fuentes alternas y nuevos puntos de vista. ${ }^{4}$

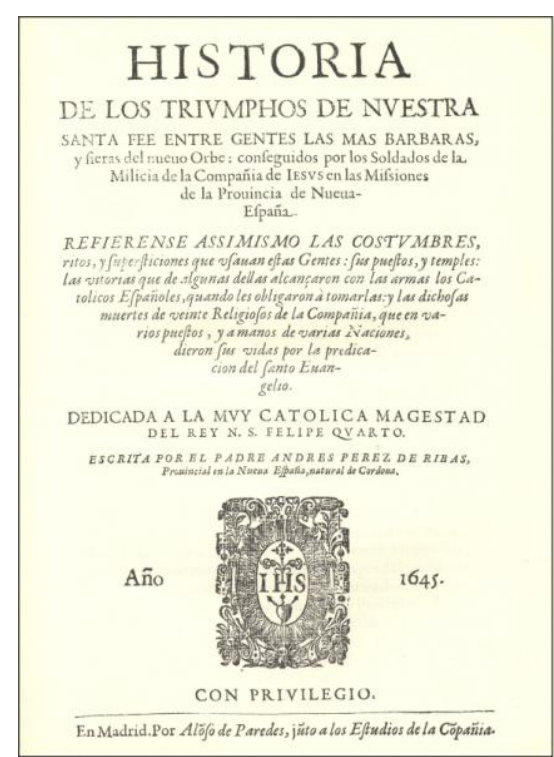

Fig. 1 Portada interior de la obra Triumphos de nuestra santa fee, de Andrés Pérez de Ribas, Madrid, 1645.

\footnotetext{
${ }^{1}$ Solo existe la biografía publicada por Cervantes, 1942, que en gran parte se sustenta en la nota biográfica que sobre Hernando de Villafañe encontramos en la obra del misionero jesuita y cronista de la orden Andrés Pérez de Ribas.

${ }^{2}$ Un panorama general de la historia del noroeste novohispano que condensa la historiografía clásica sobre los jesuitas lo tenemos en Ortega, 1993, quien se encargó de formular una regionalización que incluye una "subregión misional". Otros ejemplos los tenemos en Gómez, 2006: 49-73 y particularmente sobre la superioridad jesuítica ante sus misiones en Rodríguez, 2013: 13-41.

${ }^{3}$ La edición en español de la obra de Herbert Eugene Bolton, Confines de la cristiandad (2001) pone de manifiesto el gran interés por Kino en diversas instituciones mexicanas, y la pasión que desató el personaje en el siglo XX.

${ }^{4}$ Pérez de Ribas, 1992: 349-357.
} 


\section{Misionar en Indias, de León (Castilla la Vieja) a Pátzcuaro}

Andrés Pérez de Ribas, tuvo oportunidad de conocerlo muy bien a pesar de haber llegado una década después a la misión de Sinaloa. Cuenta que Villafañe nació en León, en Castilla la Vieja, siendo hijo de padres nobles ${ }^{5}$ que desde su niñez dispusieron que estudiase latinidad y virtud, pasando en su juventud a la Universidad de Salamanca donde inició los cursos en derecho. ${ }^{6}$

Sin embargo el mismo testimonio del cronista jesuita consigna que Villafañe no permaneció en la Universidad ya que decidió ingresar a la Compañía de Jesús el año de 1580, a los veinte años de edad, cursando los estudios de filosofía en su ciudad natal, bajo el cuidado del padre Luis de la Puente. El "Catálogo de sujetos de la provincia de México 1555-1604" ofrece información puntual: "El padre Villafaña hizo los votos de los dos años, el mes de mayo de 1581, en la provincia de Castilla, en el collegio de León, a la missa del padre Estevan de Ojeda". ${ }^{7}$

Por su parte, Félix Zubillaga consigna en $\mathrm{Mo}$ numenta Mexicana que Villafañe debió hacer sus votos por el mes de mayo de 1581. Su paso a la Nueva España se realizó junto con otros 17 jesuitas que encabezaba el padre Francisco Vaez, quien retornaba de Roma habiendo ejercido como procurador de México. La travesía se realizó el verano de 1584 , habiendo salido de Cádiz el 26 de junio ${ }^{8}$ y llegado al puerto de Veracruz el 10 de septiembre ${ }^{9}$ y a la ciudad de México el 27 de octubre. ${ }^{10}$

Por entonces la Provincia Mexicana de la Compañía de Jesús se encontraba en fase de preparación respecto de su incursión de forma permanente entre los grupos indígenas, sobre lo que insistía en

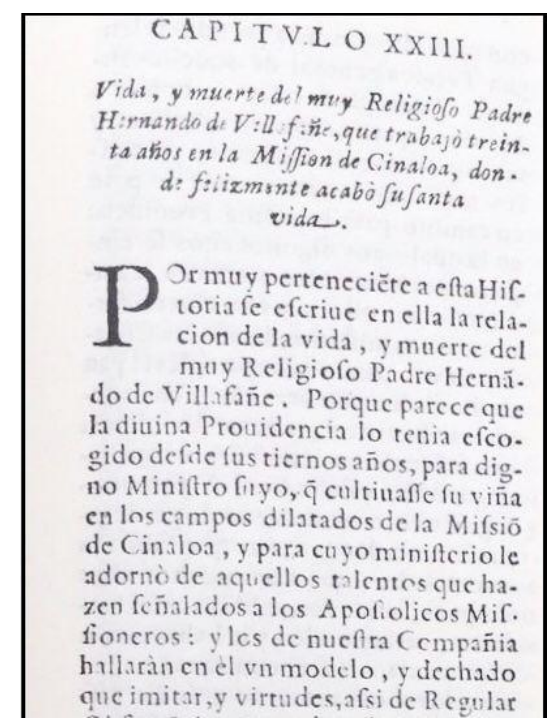

Fig. 2 Detalle de la biografía de Hernando de Villafañe en Triumphos de nuestra santa fee, p. 349.

\footnotetext{
${ }^{5}$ Desde nuestro punto de vista el tema de la nobleza de Hernando de Villafañe se trata de uno de los aspectos menos conocidos del jesuita. Sin embargo, entre los escasos referentes con que contamos actualmente debemos anotar que en el proceso de recepción en León del cuerpo de San Marcelo en el que participó Fernando el Católico el 29 de marzo de 1493 se registró que los ciudadanos regidores que participaron fueron Juan de Villamizar y Alonso Vaca, Alonso de Villafañe El Viejo y sus sobrinos Pedro y Gonzalo de Villafañe, además de García de Quirós. Posteriormente se consigna el haber sido enviado a cortes el también regidor de León Fernando de Villafañe, el 5 de mayo de 1521, sin duda un ancestro directo del jesuita que ahora investigamos, García de la Foz, 2002 [1867]: 71-72. Asimismo llama nuestra atención que casi un siglo después de Hernando, una rama de la familia Villafañe pasó al virreinato del Río de la Plata ejerciendo importantes cargos de autoridad, siendo uno de sus descendientes ya avanzado el siglo XVIII un jesuita expulso (Lovay y Page, 2013: 155-169).

${ }^{6}$ Pérez de Ribas, 1992: 349 y "Catálogo de la Provincia de Nueva España. Año 1585”, en Zubillaga, 1959: 747.

7 “Catálogo de sujetos de la provincia de México 1555-1604”, en Félix Zubillaga, 1959: 578.

8 “Gastos hechos por los padres Mendoza, Váez y Compañeros para el viaje a Nueva España”, en Zubillaga, 1959: 372-374.

${ }^{9}$ Gutiérrez, 1975: 718.

10 “Catálogo de sujetos de la provincia de México 1555-1604”, en Zubillaga, 1968: 578.
} 
sus instrucciones el general Claudio Aquaviva, ${ }^{11}$ por lo que se habían establecido en Tepotzotlán y Pátzcuaro los centros para el aprendizaje de las lenguas náhuatl, otomí y tarasco. $^{12}$

Villafañe pasó a Michoacán para aprender el tarasco, al igual que su amigo y coterráneo Gonzalo de Tapia que había sido compañero de viaje. Se ha destacado el paso inmediato a la residencia de la Compañía de Jesús en Pátzcuaro, sin haber terminado sus estudios. De hecho solo contaba con votos simples, al haber realizado hasta entonces solo tres años de filosofía y uno de teología. Lo cierto es que aquella ocasión brindó al joven Villafañe la oportunidad de conocer los métodos misionales del padre Juan Ferro, un jesuita que trabajó entre los tarascos por muchos años, realizando misiones temporales en el entorno cercano, como en la Tierra Caliente, Guanajuato y los Chichimecas y entre cuyas estrategias encontramos la elaboración de catecismos en lengua tarasca. ${ }^{13}$

Por lo demás, es significativo en cuanto a su formación, que en el "Catálogo de la provincia de Nueva España, año de 1585", Villafañe aparece en el listado del Colegio de México, lo que significa que los superiores estuvieron atentos a sus estudios que se alternaron con el aprendizaje de la lengua y el trabajo en misiones. ${ }^{14}$

La "Relación de la residencia de Pátzcuaro", realizada en 1586 pero que refiere a la situación de 1585 consigna la llegada de nuevos padres que "rápidamente han aprendido" como lo fueron Cristóbal de Bravo y Gonzalo de Tapia, quienes predicaban y confesaban en tarasco, a la vez que los aún estudiantes Hernando de Villafañe y Gerónimo Ramírez, quienes a pesar de haber aprendido la lengua,

no pudieron confesar por no ser sacerdotes [aunque], no dexaron de ayudar con pláticas y doctrinas. Agora están acabando de oyr su teología en México, para poder volver después a ayudar a tanta mies como hay aquí. Vinieron tan aficionados a estos indios, que se les hace muy largo el tiempo de los estudios: tanto es el deseo que tienen de volver de allá. ${ }^{15}$

Lo cierto es que aún pasaron cinco años para que fuese sacerdote, de forma que a la vez que continuaba su aprendizaje y práctica de la lengua tarasca y que realizaba misiones, alternaba sus estudios en el colegio de México. Sus exámenes de epístola, evangelio y misa se realizaron con éxito entre abril y mayo de 1590 , ordenándose ante el obispo de Oaxaca Fr. Bartolomé de Ledezma. ${ }^{16}$

Una de las primeras encomiendas de mando que recibió el padre Villafañe fue la de dirigir el colegio de Valladolid. Estaba allí como rector el año de 1592, época en que con 32 años de edad el "Catálogo de sujetos de la provincia de Nueva España" destacaba su "flaca salud". Como sea, encontramos una constante en su trayectoria en la compañía que consistía en su falta de apego al mando de otros. En este caso pidió con éxito

\footnotetext{
11 "El padre Claudio Aquaviva, general, al padre Antonio de Mendoza, provincial", Roma, 15 de marzo de 1584, en Zubillaga, 1959: 270-280.

${ }^{12}$ López, 2013: 15-39.

${ }^{13}$ Alegre, 1956: 306 y 577.

14 “Catálogo de la Provincia de Nueva España. Año 1585”, en Zubillaga, 1959: 747.

15 “Relación de la residencia de Pátzcuaro [principios de 1586]”, en Zubillaga, 1968: 51-53.

16 “Catálogo de sujetos de la provincia de México 1555-1604”, en Zubillaga, 1968: 601.
} 
al general ser exonerado de esta responsabilidad en virtud de sus constantes jaquecas. ${ }^{17}$ No obstante poco después tenemos que Hernando de Villafañe recibió la encomienda para dirigir el trabajo en la residencia de Pátzcuaro, que fue el centro en el que se formaron varios de los más notables misioneros que ejercieron en el norte novohispano. Pérez de Ribas describe así aquella época:

Estando en este colegio de Pátzcuaro, fue elegido de los superiores por rector dél, donde exerció su oficio con mucha prudencia y vigilancia, atendiendo a un tiempo, así al provecho de los súbditos, como el de los muchos españoles que alli hay, y naturales indios, ayudando a unos y a otros con sus frecuentes sermones en sus lenguas. ${ }^{18}$

De hecho ahí se encontraba cuando aconteció la muerte del padre Gonzalo de Tapia, quien era el superior de Sinaloa, a manos de Nacabeba, el 11 de julio de 1594. Las noticias se esparcieron por la Provincia Mexicana de la Compañía de Jesús y particularmente uno de los testimonios provino de los indígenas tarascos que trabajaban en las minas de Topia. Ellos realizaron una carta en su propia lengua que enviaron al Colegio de Pátzcuaro en donde lamentaban la muerte del misionero jesuita que se había formado en sus tierras y de quien habían recibido doctrina en la sierra. ${ }^{19}$ No pasó mucho tiempo para cuando Hernando de Villafañe hizo llegar al provincial la solicitud por "suplir" a Tapia. El pasaje lo narra Pérez de Ribas como el momento clave en el que el jesuita opta por ir a la misión de Sinaloa. Sin embargo, el "Catálogo de (jesuitas en) Nueva España", fechado el 29 de diciembre de 1595 lo ubicaba aún en el Colegio de Pátzcuaro, quedando sin precisar el momento de su partida. ${ }^{20}$

\section{Misionero en Sinaloa entre los guasaves}

Villafañe llegó a la provincia de Sinaloa en 1596, donde recibió la encomienda de evangelizar a los grupos indígenas del bajo río Petatlán. No fue el primer jesuita que había llegado a aquellas tierras, al menos tres de los fundadores de la misión de Sinaloa realizaron algún tipo de incursión.

El primero fue Martín Pérez en 1591, quien realizó una entrada de reconocimiento de los grupos indígenas localizados río abajo de la villa de San Felipe y Santiago de Sinaloa. Consigna entre ellos a los bamoas (que eran de origen pima o nebome), guasaves, sisimicaris y ures, además de otras rancherías dispersas hasta la desembocadura. De este primer encuentro de los guasaves con los misioneros queda constancia en Andrés Pérez de Ribas quien se apoya en una carta de uno de los padres, que de acuerdo a la lógica sería el propio Martín Pérez, donde se consigna que se realizaron los primeros bautizos de párvulos:

Hize una salida por pueblos de gentiles, cuya lengua aún no sabía, en llegando me ofrecieron con muy buena y alegre voluntad más de doscientos y cincuenta niños, para que los bautizasse, como lo hize; y para poder ayudar a los adultos, in extrema vel gravi necesitase, hize un catecismo breve en su lengua por medio

\footnotetext{
17 "IHS. Catálogo primero del año mil y quinientos y noventa y dos, de los padres y hermanos de la Compañía de Jesús, desta provincia de la Nueva España”, en Zubillaga, 1968: 375 y 385.

18 Pérez de Ribas, 1992: 350.

${ }^{19}$ Ibíd.: 136-137.

20 “Catálogo de Nueva España. México 29 de diciembre de 1595”, Zubillaga, 1973: 513.
} 
de un intérprete que la sabía y con cuatro palabras que les dezía de nuestro señor y las más por el papel, era grande la suspensión y atención con que las oían". 21
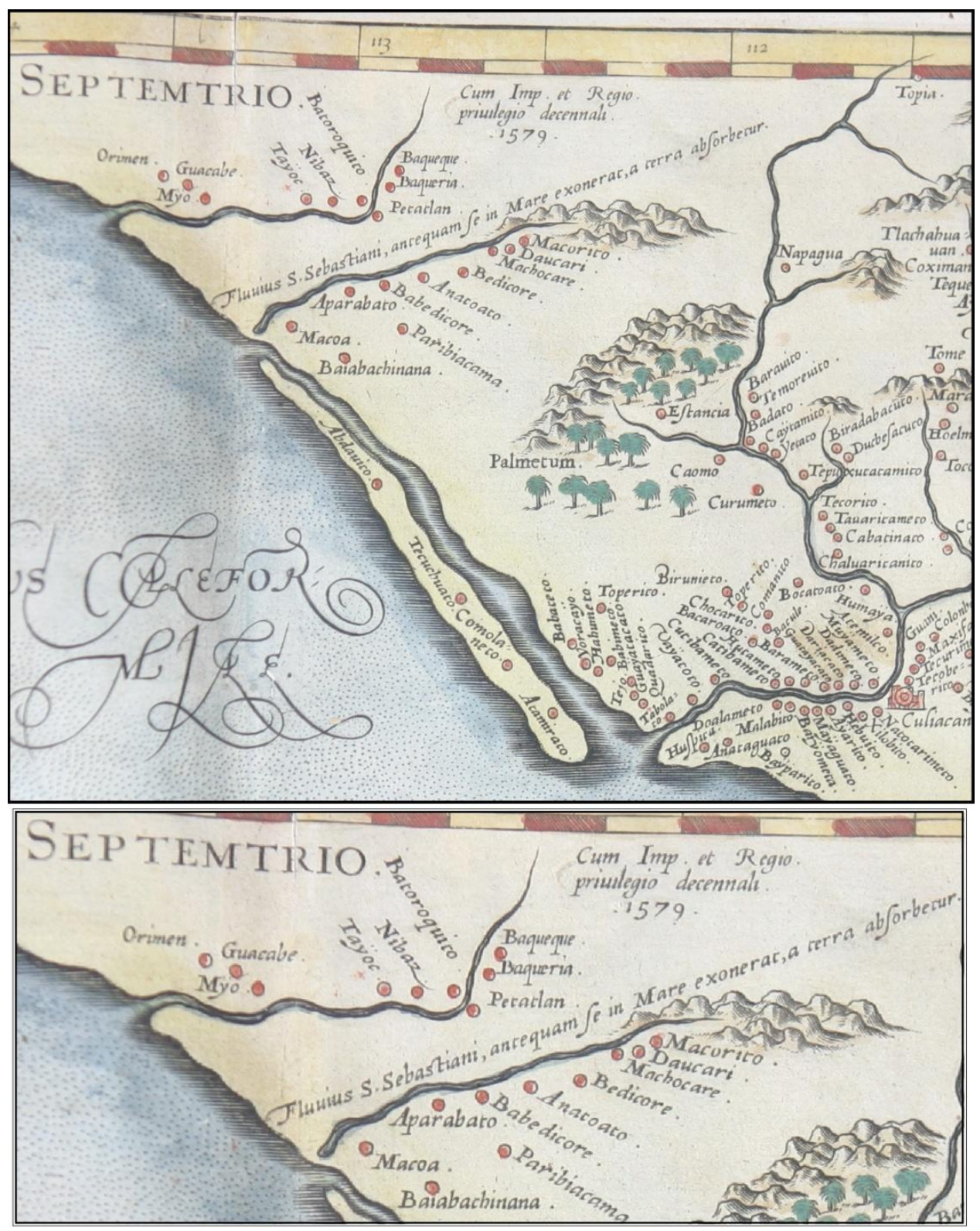

Mapa 1 "Culiacanae, Americae Regionis Descriptio, de Abraham Ortelio, en Theatrum Orbis Terrarum, Antverpiae, exoficina plantiniana, 1579. Detalle de pueblos del río Petatlán o Sinaloa.

\footnotetext{
${ }^{21}$ Pérez de Ribas, 1992: 43. Como se desprende del testimonio del padre Martín Pérez, en el bajo río Petatlán había distintas parcialidades indígenas con sus nombres específicos. Sin embargo, pasados los años de la primera evangelización los nombres originarios se perdieron y de hecho solo permanecieron como misiones los pueblos de Guasave y Tamazula, en los que fue concentrada la antigua población dispersa. Sobre ambos pueblos ver una evaluación moderna de sus templos e información documental histórica en Santos, 2015: 73-87. Fueron sus vecinos al oriente remontando el curso del río los indígenas de las misiones de Nío y Bamoa.
} 
En el discurso del padre citado por Pérez de Ribas tenemos aún la ausencia de algún tipo de conflicto, siempre presente en el encuentro y choque de culturas. Digamos que es $s u$ testimonio, que consigna algunas de las estrategias misionales como es la exploración de naciones que "piden" su presencia, el uso de intérpretes y la elaboración de catecismo en lengua nativa. La omisión se trataba de una estrategia más que buscaba el apoyo para la empresa misionera, tanto al interior de la propia orden, como el apoyo de las autoridades de la monarquía ante una mies tan prometedora.

El propio Martín Pérez en su "Relación de la provincia de Nuestra Señora de Sinaloa" expresa el listado de pueblos del bajo río Petatlán:

Más debajo de este río, donde habrá 400 indios de arco y flecha, a una legua de allí está Guasave, y más abajo Sisinicare, Chichile, Pohui, Baborojo, Ure, Tohigo, Pocohohuaca, en los cuales hay, según se dicen, más de 4000 almas de una lengua, y los toroguacos están a la orilla del mar. ${ }^{22}$

Un segundo momento, propiamente entre los guasaves lo tenemos con el padre Juan Bautista Velasco, cuya presencia se consigna en el contexto de una epidemia de viruela y sarampión del año 1594 que se había extendido desde la villa de San Felipe y Santiago y su entorno cercano, donde ya se había establecido la presencia de los jesuitas, hasta los demás pueblos aún "gentiles" del entorno. Así, su labor tuvo más bien la tarea de bautizar niños enfermos consignando hasta 250 párvulos que fallecieron en pocos días además de medio centenar de adultos. No obstante gracias a ambos testimonios vamos ubicando en el territorio a los distintos grupos que los jesuitas identificaban como "guasaves", que se encontraban en el centro mismo de la misión de Sinaloa.

El tercer jesuita que tuvo permanencia en el bajo río Petatlán fue Hernando de Santarén. A él le correspondieron ya momentos de conflicto ante la política jesuítica que buscaba la transformación de la tradicional forma de vida, la concentración de los indígenas de sus antiguas rancherías en pueblos más grandes y la eliminación de todas aquellas manifestaciones culturales que los religiosos consideraban como idolatrías y que por tanto entraban en conflicto con el proceso de evangelización que se había emprendido. Durante el primer lustro de contacto con los jesuitas hubo una tensa calma y un frecuente estado de insurrección indígenas. Fue uno de los primeros lugares donde se dio el choque con las armas españolas, debido a la conformación en 1596 del presidio de Sinaloa bajo el mando del teniente general Alonso Díaz que reprimió a sangre y fuego a los líderes nativos. En este contexto se dio la llegada de Hernando de Villafañe, quien entre 1596 y 1598 acompañó en el bajo río Sinaloa a Hernando de Santarén. Andrés Pérez de Ribas consigna puntualmente cómo la pacificación del territorio por la vía de las armas abrió la posibilidad para:

asentar de paz con algunos pueblos comarcanos, que todavía andaban inquietos como fueron los de Nío y Vacayoe, que distan della cinco leguas, en que auia como quinientas familias, y tres leguas más abaxo los pueblos de la nación Vacave, que poblaban hasta la mar y boca del río: y por la costa adelante, que era mucha su gente, y tenía de tres a quatromil indios de arco y flecha. ${ }^{23}$

El aprendizaje de la lengua Guasave "que corría por todas las marismas de Sinaloa" fue una de las primeras acciones del jesuita. De hecho, en tiempos en que se encon-

\footnotetext{
${ }^{22}$ González y Anzúrez, 1996: 198.

${ }^{23}$ Pérez de Ribas, 1992: 58-59.
} 
traban ambos jesuitas allí echaron mano para doctrinar a los indios de una india cristiana y ladina que había sido antes esclava de los españoles de Culiacán. La insurrección de 1598 provocó una salida temporal de ambos padres y a partir de entonces, Hernando de Santarén pasó a la misión entre los acaxees, mientras que Villafañe continuó su labor entre los guasaves desarrollando así algunas acciones que, de acuerdo con Pérez de Ribas se convirtieron en el referente de la acción misionera del resto de la misión de Sina$\operatorname{loa}^{24}$ que ya en 1605 se abría paso entre los pobladores del río Zuaque.

Consignaremos asimismo que la profesión definitiva de cuatro votos la obtuvo ya estando en la misión de Sinaloa poco antes de culminar el siglo XVI. El visto bueno lo emitió el mismo general Claudio Acquaviva, sin embargo correspondió otorgarlo a quien era el superior directo, en este caso el padre Martín Pérez, titular de la residencia de Sinaloa, lo cual fue una concesión especial vigente en la Provincia Mexicana de la orden jesuita, por encontrarse Hernando de Villafañe realizando su trabajo de evangelización en "provincia muy distante".

La nueva y definitiva pacificación entre los guasaves sucedió en 1599 y corrió a cargo del nuevo capitán del presidio de Sinaloa Diego Martínez de Hurdaide, quien castigó a los alzados a la vez que emprendió la estrategia de perdonar al cacique principal que fue bautizado como don Pablo de Velásquez, que en adelante fue interlocutor para la cooperación de los guasaves en la evangelización del propio río. ${ }^{26}$

Tenemos información de la misión entre los guasaves en la Carta anua de la provincia de México de 1603, que nos permite ver cómo es que se fue asentando la población indígena de acuerdo al planteamiento de los jesuitas y particularmente del padre Villafañe quien siendo superior de la residencia de Sinaloa mantuvo su actividad misionera en el bajo río Petatlán. Esta circunstancia de rol dual de una autoridad misionera fue una característica que perduró en Sinaloa en virtud que era un establecimiento en tierra de misiones y de que no había en realidad suficientes jesuitas para que algunos se encargaran de forma exclusiva del mando desde el punto central. De hecho en ésta carta anua se consignaba que el partido de Guasave era el mayor de todos los dependientes de Sinaloa en aquel momento, y que en los pueblos había poco más de seiscientos vecinos, todos los cuales habían sido casados y que hablaban una misma lengua, la guasave existiendo simultáneamente un gran número de población indígena dispersa a lo largo de la costa, con sus propios asentamientos. ${ }^{27}$

El testimonio se describe así:

De los indios que al presente se doctrinan, algunos estaban juntos en sus pueblos. Otros, como son los marítimos, se handaban por los montes y esteros del mar, buscando qué comer, y mudando a menudo ranchos. No sembraban ni tenían más casas que unas esterillas, que les servían, de día, de toldo, y de noche, de cama. Y no tenían otras ropas que las redes con que pescaban. Llaman, por acá, a estos los ratoneros, porque se sustentaban de ratones, culebras, raízes, lagartijas y otras sabandijas y frutillas del campo, y de pescado y mariscos, con que pasaban su vida holgazana y brutal. Estos también hacían ollas y otros va-

\footnotetext{
${ }^{24}$ Ibid.: 353.

25 “Catálogo de sujetos de la provincia de México 1555-1604”, en Zubillaga, 1968: 642.

${ }^{26}$ Pérez de Ribas, 1992: 81-82.

27 “Carta anua de la provincia de México. México, 5 de mayo de 1603”, en Rodríguez, 1991: 152.
} 
rios vasos de barro y redesillas. Y con esto y algún pescado y camarón, venían a los pueblos a rescatar maíz. Destos se han recojido, por industria de un padre, muy buena cantidad, y anse asentado en sus pueblos, principalmente en el de Tamazula, que es de los mayores que hay por acá, cerca de la mar, donde gozan de mucho pescado y ostiones. Han hecho casas y ánseles dado tierras y siembran mucho maíz. ${ }^{28}$

Se trata de una descripción amplia y rica en detalles. En primer lugar porque se destaca la convivencia en este espacio del territorio de indígenas agricultores con otros que, aunque eran "marítimos", es decir habitantes del litoral en la desembocadura del río, esteros, islas y penínsulas, también realizaban actividades que les ofrecía la susbsistencia, principalmente la pesca, aunque también la recolección que por ser una actividad de indígenas libres aparece a ojos del jesuita como algo despreciable. No obstante, pasados los comentarios peyorativos se les reconocen otros tipos de actividades que le daban complementariedad a su forma de vida, como es la alfarería y la elaboración de redes de pesca, todo lo cual les brindaba la oportunidad de realizar comercio mediante el intercambio de sus bienes por maíz.

De hecho en general entre los cahitas, territorio que comprendía la franja costera desde el río Mocorito hasta el Yaqui se ha encontrado bastante complementariedad entre la actividad económica de los diversos grupos que lo habitaban. ${ }^{29}$

El punto de vista del jesuita es muy claro, debido a que para ellos la vida en libertad, dispersos por los montes y "cazando sabandijas" se contraponía a la vida en policía, bajo campanas y asentados en los pueblos. Por eso el énfasis en el arraigo que busca dárseles en el pueblo de Tamazula que es el asentamiento que a la postre concentraría a estos habitantes originalmente llamados marítimos y a quienes se les asignaron tierras como una forma de buscar su permanencia. Cabe además destacar que esta es una de las primeras menciones sobre el pueblo de Tamazula y que, como se puede apreciar, fue el asiento de los indígenas provenientes de rancherías del entorno. La misma fuente consigna otro, Tzitzinicari sobre el que no hay más referencias. ${ }^{30}$

La edificación de los templos misionales fue una de las acciones de mayor impacto por las diversas implicaciones que tenía entre la población nativa. Pérez de Ribas lo narra de forma extensa:

Estando en este grado las cosas, el padre Hernando de Villafañe que tenía a su cargo la grande nación de Guasave, habiéndose ya desembarazado de bautismos en los pueblos, trató de fabricar iglesias de asiento y capaces para la mucha gente que se había bautizado. Obra era ésta, nueva y nunca vista en aquella tierra, y de trabajo para los indios; pero como los de esta nación eran de más blando natural y aplicación al trabajo, que otras, y por ser estas fábricas de gran importancia para hacer asiento en los pueblos los redujo el padre Villafa$\tilde{n}$, a hacer iglesias, aunque de adobes; pero fuesen bien cubiertas de asoteas y terrados, y libres de los incendios de que están sujetas las de madera y paja. Pusieron manos a la obra; hicieron en los tres pueblos principales mucha cantidad de adobes. Comenzaron a levantar las paredes, y, mientras más iban creciendo,

\footnotetext{
${ }^{28}$ Ibídem.

${ }^{29}$ Carpenter, 1999: 119-128.

30 “Carta Anua de la provincia de México, México, 5 de mayo de 1603”, en Rodríguez, 1991: 153.
} 


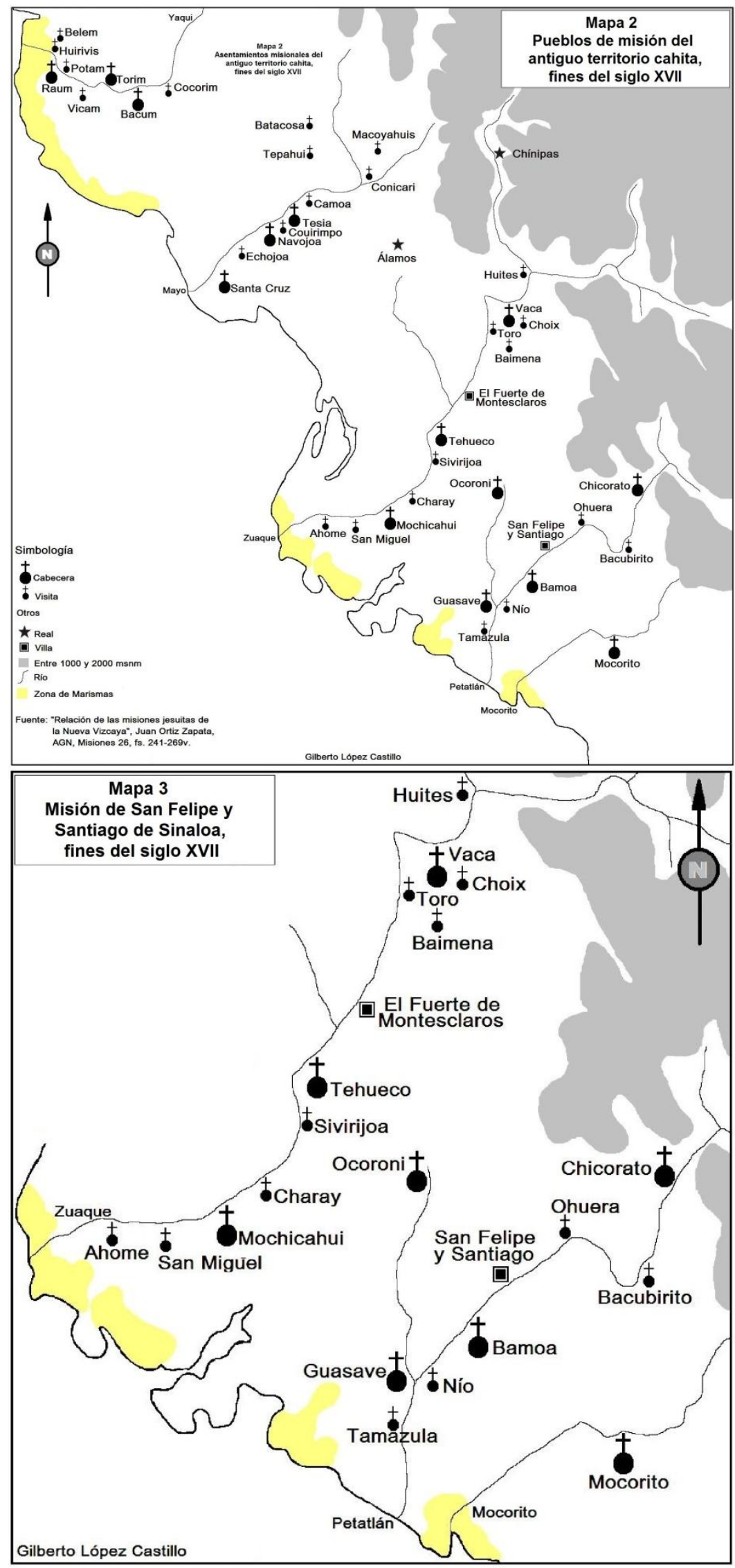

Mapas 2 y 3 Pueblos de misión del antiguo territorio cahita, fines del siglo XVII. Misión de San Felipe y Santiago de Sinaloa, fines del siglo XVII. 
crecía en ellos el deseo de ver acabada la obra; cortaron y trajeron a hombros (porque ellos son valientes en estas cargas) y labraron cantidad de árboles para su enmaderamiento y quedaron hechas tres muy grandes iglesias que, aunque no eran de cantería, salieron muy vistosas en aquellas tierras; por que el padre Hernando de Villafañe procuró adornarlas, blanqueándolas y pintándolas, con los colores que allí se hallan. ${ }^{31}$

Era distinta porque se trataba de un nuevo tipo de construcciones que por su monumentalidad distaba de las chozas tradicionales, porque se introducían nuevos elementos a la obra como son los adobes de tierra, porque se tenía que organizar la mano de obra indígena para un tipo de trabajo antes inexistente y también porque en el modelo jesuítico de evangelización se trataba del escenario principal desde donde ahora se buscaría establecer el control de la vida de los indígenas. ${ }^{32}$

A Villafañe le toco también aprender en carne propia respecto de la construcción de los templos al ser éstos destruidos por la inundación de 1604, apenas de haber sido culminados. $^{33}$ (Fig. 3 y 4) Prueba y error que ameritó nueva construcción y que, evidentemente no solo tuvo que pagar él, quien se decía actuaba como sobrestante, sino también los propios indígenas del bajo Petatlán que después de las rebeliones de la última década del siglo XVI fueron señalados como grandes aliados de los españoles.

Las actividades en estos pueblos, propiamente, de misión, son algunas de las que hemos mencionado en los párrafos anteriores, y los padres buscaban de forma sistemática mantener el orden de acuerdo a su esquema moral y religioso donde el matrimonio jugaba un rol importante, dado que la costumbre indígena era muy liberal al respecto, pudiendo anteriormente casarse muy fácilmente mujeres y hombres con otros de acuerdo a su libre albedrío. El consumo de alcohol entre los guasaves también fue restringido en tiempos de Villafañe que, dado el caso, contaban para su apoyo con los soldados del presidio de Sinaloa, de tal forma que la elaboración de vino pasó a ser una tarea clandestina que en caso de ser descubierta era castigada.

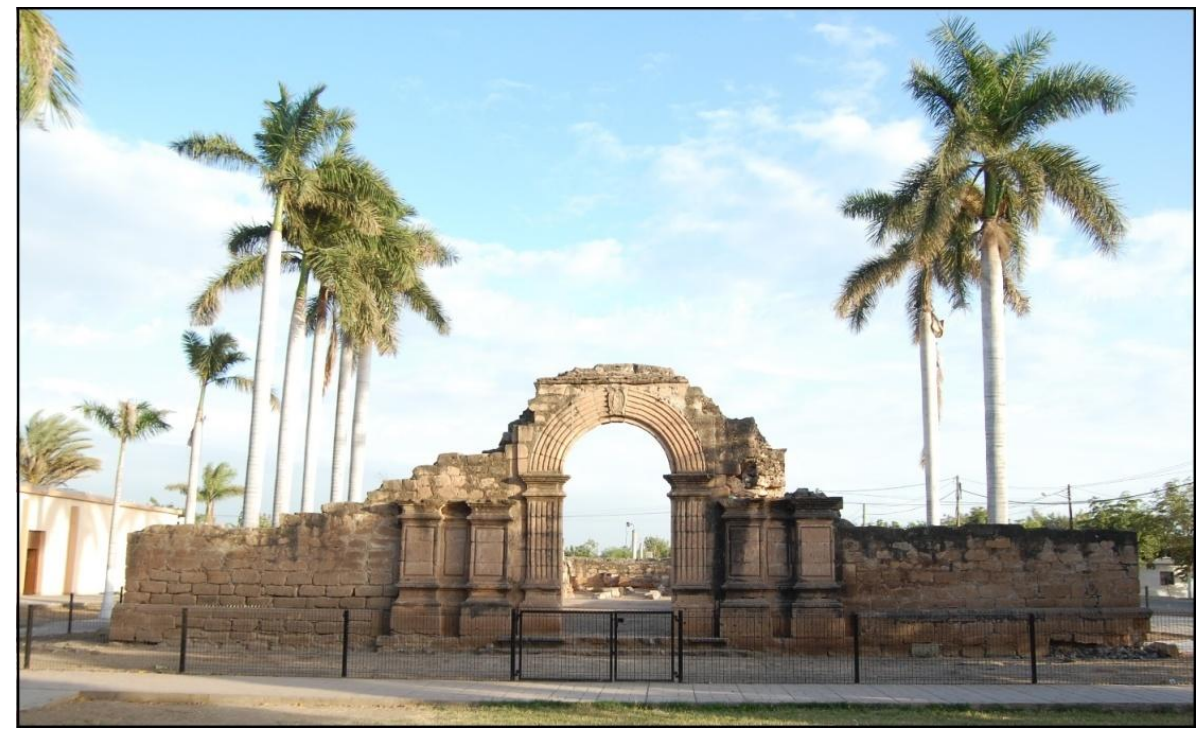

Fig. 3 Vestigios de la misión de Nío (Autor: Víctor Joel Santos Ramírez).

\footnotetext{
${ }^{31}$ Pérez de Ribas, 1992: 97-98.

${ }^{32}$ Sobre los vestigios actuales de la arquitectura misional ver Santos, 2015.

${ }^{33}$ Pérez de Ribas, 1992: 97-98.
} 

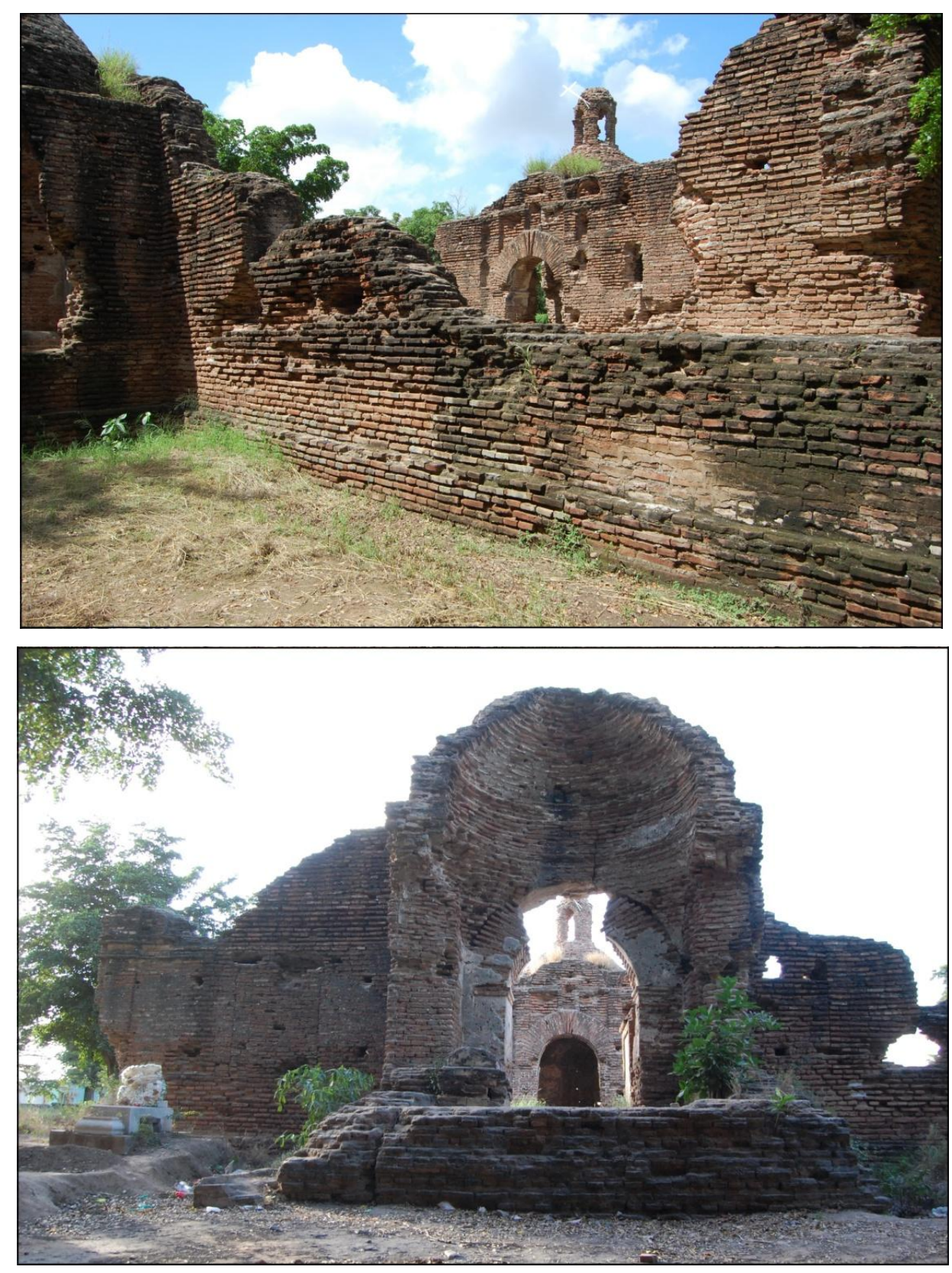

Fig. 4 Vestigios de la Misión de Nío Pueblo Viejo (Autor: Víctor Joel Santos Ramírez).

En general, nos dice Pérez de Ribas en un punto de vista que ha recogido la historiografía de forma insistente, los aspectos desarrollados por Villafañe en su misión entre los guasaves se erigieron en punto de referencia para los demás misioneros de la provincia de Sinaloa, quienes se dirigían allí para ver cómo obrar en sus distintos partidos. Otro historiador jesuita, Gerard Decorme, ya en el siglo XX ratifica el punto de vista de la obra de Villafañe en el sentido de la emulación de su trabajo que, corría fama, realizaban los demás jesuitas en misión. ${ }^{34}$

Es importante relacionar este momento histórico, es decir, la primera construcción de las iglesias misionales con los relatos rescatados por el padre Juan Ortiz Zapata

\footnotetext{
34 “En los 30 años que cultivó esta misión (con alguna interrupción), hizo de Guasave una cristiandad modelo, donde todos los nuevos misioneros venían a aprender la organización y cultivo de aquellas naciones", Decorme, 1941: 171-172.
} 
en 1678 en donde se refiere la tradición en que se fincó la fe de las personas en la imagen de la Vírgen de Nuestra Señora en el pueblo de Guasave. Estos relatos nos hablan de la época misional temprana, de las epidemias e inundación de los templos, es decir, al menos de la primera década del siglo XVII, momentos en que si bien Villafañe misionaba, también lo es que realizaba otras actividades alternas de gobierno, tanto en la residencia de Sinaloa, como del conjunto de misiones de la Nueva Vizcaya, lo que nos impide hablar con certeza respecto de que haya sido el protagonista, aunque por su labor específica se trata del candidato más probable. ${ }^{35}$

\section{Villafañe gestor}

La actividad de Villafañe en los pueblos de misión queda un poco desdibujada en los años posteriores, no porque no se encargara más de la misión al ras del suelo, sino porque quizá él mismo dejaba menos testimonios de asuntos que le eran cotidianos y, en todo caso, lo vemos muy activo en la planificación de actividades de la misión de Sinaloa en un sentido más amplio. Por ejemplo en algunas de las primeras comunicaciones de Claudio Acquaviva a su persona tienen que ver con el visto bueno que ofrece el prepósito general para que se funde una nueva villa al norte de San Felipe y Santiago, la cual serviría para consolidar las nuevas misiones del río Zuaque. ${ }^{36}$ Pérez de Ribas al referirse al trabajo de Villafañe como gestor destaca la estrecha relación que tuvo con las autoridades de la Nueva España tocante a la consecución de beneficios para las misiones, más aún si consideramos que muchas de las ocasiones que intercedió por la provincia de Sinaloa lo hizo en su carácter de superior de la residencia de Sinaloa. ${ }^{37}$

Hernando de Villafañe era sin duda un personaje significativo en las misiones y gracias a ejercer como superior de la residencia en distintos momentos entre $1598 \mathrm{y}$ 1613 permaneció misionando de forma intermitente entre los guasaves. Así, a la vez que se encargaba del gobierno de aquel establecimiento, realizaba visitas periódicas a los pueblos bajo su jurisdicción y palpaba directamente la situación de las misiones bajo su cargo lo que permitía un conocimiento muy puntual de la situación que posibilitaba interactuar con los superiores para que atendiesen las necesidades. En reconocimiento de su importancia específica recibió en 1611 el nombramiento de "superintendente de las misiones de Nueva Vizcaya y Cinaloa", y más tarde, en 1619, el de "superintendente general" o "visitador de las misiones de Sinaloa". 38

Pero no solo realizó su carrera en las misiones, muy poco después de ese lapso en que deja la misión de Sinaloa por encargos en los colegios centrales. En 1613 el ge-

\footnotetext{
35 "Relación de las misiones que la Compañía tiene en el Reyno y provincia de la Nueva Vizcaya, en la Nueva España, hecha el año de 1678 con ocasión de la visita general de ellas que por orden del padre provincial Thomas Altamirano hizo el padre visitador Juan Ortiz Zapata, de la misma Compañía", Archivo General de la Nación México (AGN), Misiones 26, fs 241-269v.

36 "El padre general Claudio Aquaviva al padre Hernando de Villafañe, abril de 1601", ARSI, México 02, Epistolario del General (en adelante Ep. Gen.), 1599-1638: 5v.

${ }^{37}$ Pérez de Ribas, 1992: 98-99.

38 "El padre Claudio Acquaviva, general, al padre Hernando de Villafañe", Roma, 1611, ARSI, México 02, Epp. Gen.: 146v., y "El padre Mutio Vitelleschi, general, al padre Hernando de Villafañe visitador general de Cinaloa", Roma 1619, ARSI, México 02, Epp. Gen.: 211v. Sobre la comunicación de los jesuitas con el general y particularmente de las misiones, López, 2013: 121-136.
} 
neral Aquaviva lo nombró rector del colegio de Puebla ${ }^{39}$ y quizá entre 1615 y buena parte de 1616 ejerció como rector del Colegio de México. Es decir, Villafañe tenía para ese momento la experiencia de los distintos tipos de establecimientos jesuíticos de la Provincia jesuítica Mexicana.

Era en estos casos donde no se le encontraba agusto y entonces pedía ser relevado del "mando de otros". No obstante, la autoridad central vía la regla ignaciana de la obediencia lo hacía permanecer como rector donde lo necesitase, justamente para aprovechar su experiencia en el mando

No deja de ser significativo que el paso del estatus de residencia a colegio de Sinaloa ocurrió en 1616 y el primer rector del colegio lo fue, justamente Hernando de Villafañe. ${ }^{40}$ Tenemos aquí una semejanza en las trayectorias de Andrés Pérez de Ribas y Hernando de Villafañe que fueron, cada uno en su momento, dos referentes de las misiones de Sinaloa y que aun estando en puestos superiores seguían buscando respaldo a la empresa misional.

\section{Procurador a Roma y últimos años}

Había pasado un cuarto de siglo desde la llegada de la Compañía a Sinaloa y algunos de los miembros de aquella primera generación de jesuitas habían fallecido, mientras que otros simplemente habían pasado a establecimientos centrales de la orden en la Provincia Mexicana. En ese contexto, la participación de Villafañe como rector del colegio de Sinaloa en la Congregación de la provincia Mexicana de la Compañía de Jesús, reunida el 2 de noviembre de 1619 en el colegio máximo tuvo entre sus principales postulados la necesidad de suplir con nuevos jesuitas a las misiones. El liderazgo de Villafañe fue tal que logró ser nombrado como Procurador a Roma y Madrid, junto con el rector del colegio de México, padre Laurencio Adame. ${ }^{41} \mathrm{Y}$ ahí tenemos, de regreso al jesuita al Viejo Mundo el año de 1620, treinta y cinco años después de su paso a Nueva España. Era un veterano de la provincia Mexicana de la Compañía de Jesús y su viaje significó el encuentro con el nuevo general, Mutio Vitelleschi que por demás era quien lo había nombrado rector en una de las primeras acciones de su gobierno el año de 1616.

De hecho la primera solicitud de la Congregación provincial a Roma de la que era portador Villafañe era el fortalecimiento con más sacerdotes, principalmente para misiones, ello debido a tres razones, una el crecimiento del área misional, dos la muerte natural de algunos de los primeros padres y finalmente por la muerte de seis jesuitas en la rebelión tepehuana. ${ }^{42}$

\footnotetext{
39 "El padre Claudio Acquaviva, general, al padre Rodrigo de Cabredo, provincial, nombramientos de autoridades", Roma, 1613, ARSI, México 02, Epp. Gen., f. 159.

40 "El padre Mutio Vitelleschi, general, al padre Rodrigo Cabredo, provincial de México, 1616, asiento de los nuevos superiores de las casas y colegios de esa provincia" (rector del colegio incoado de Sinaloa Hernando de Villafañe, para todos los cuales se envían patentes), ARSI, México 02, Epp. Gen.: 180v.

41 "Nona congregación provincial. Memorial de lo que se pide a nuestro padre, y se a de tratar con su paternidad, por orden del padre provincial Nicolás de Arnaya", en Alegre, 1958: 640-642.

${ }^{42}$ El texto de la "Nona congregación provincial" se complementa con el "Memorial del padre Arnaya, provincial de la Compañía de Jesús de Nueva España, y sus consultores, en 12 de mayo de 1620, para nuestro padre general", en Alegre, 1958: 642-643.
} 
Nos quedamos con el testimonio que sobre la visita del padre Villafañe a Roma nos da el propio general Mutio Vitelleschi al expresarlo al provincial de México, el padre Nicolás de Arnaya:

El padre Hernando de Villafañe, procurador de esa provincia y su compañero llegaron acá, gracias a Dios con la salud que yo deseaba, hele oydo muy despacio con muestra (....) zelo mío y he experimentado todo lo bien que él me dice, $V R$ tiene muchas noticias de las cosas de la provincia y especialmente de las missiones como quien las ha gobernado tantos años, en todo nos ha informado con grande fidelidad y verdad y nos (h)emos edificado con su mucha religión y ejemplo y con la buena relación que nos ha dado del bien que los hijos de la Compañía que están en esa provincia atienden a su aprovechamiento espiritual y juntamente a ayudar a la salvación de los próximos. Elévelo el señor adelante como se lo suplico. ${ }^{43}$

También vale la pena destacar dos aspectos de este viaje, el primero tiene que ver con la repetición del ciclo por el que el mismo Villafañe había cruzado el Atlántico en 1585, es decir, siendo parte de un grupo de jesuitas españoles que acompañaban en su regreso al padre Vaez. En su turno como Procurador a Roma y Madrid Villafañe también volvió con un importante número de jesuitas -24 aprobados por el Consejo de Indias- para fortalecer las misiones. ${ }^{44} \mathrm{El}$ otro tema que ha destacado Pérez de Ribas, así como la historiografía sobre Villafañe nos remite a los esfuerzos que realizó para poder impartir la misa durante el largo viaje de navegación entre el Viejo y el Nuevo Mundo, habiendo sido el primero en lograrlo y estableciendo norma para el futuro. ${ }^{45}$

La etapa final en la vida de Hernando de Villafañe la ocupan los años posteriores a su regreso de Roma en que volvió a dirigir el colegio de Sinaloa. Destaca aquí su participación en la ciudad de México en la congregación provincial, viaje que tuvo que realizar nuevamente desde Sinaloa a pesar de su enfermedad de gota. ${ }^{46}$

Otras actividades que le llevaron muchos años tienen que ver con el nombramiento de comisario del Santo Oficio que ejerció tanto en Sinaloa como en la provincia de Culiacán. De hecho fue el principal pesquisidor en la investigación sobre juegos de azar y brujería en la villa de San Miguel de Culiacán. ${ }^{47}$ La década de 1620 vio asimismo cómo el Villafañe emprendedor era censurado por los mismos compañeros del colegio, quienes llamaban la atención del general respecto de la infraestructura hidráulica que de su mandato se construía para regar las tierras del colegio de Sinaloa. Los tiempos habían cambiado y ya viejo se le miraba no solo con admiración, sino también con recelo de que fuese a terminar con los recursos y propiamente a endeudar al colegio. ${ }^{48}$

No obstante perduró el resto de su vida entre el cargo de rector de Sinaloa que recibió en su retorno de Roma y la práctica misionera en la misión de Guasave, a la que

\footnotetext{
43 “Carta del general Mutio Vitelleschi al Provincial de México, ARSI, Mex. 02, Ep. Gen. f. 241v.

44 "El padre Mutio Vitelleschi, general, al padre Hernando de Villafañe, procurador,", preguntando sobre su feliz regreso a México junto con los 24 jesuitas aprobados por el Consejo, con una apostilla donde se consigna la llegada, ARSI, Mex. 02, Ep. Gen. , 1623, fs 283v-284.

${ }^{45}$ Pérez de Ribas, 1992: 355.

${ }^{46}$ Ibíd., 1992: 354.

${ }^{47}$ Castro y Cuevas, 1992.

48 "El padre Mutio Vitelleschi, general, al padre Juan Lorencio, provincial,", sobre que remedie lo que trata de hacer el padre Villafañe, ARSI, Mex. 02, Ep. Gen. , 1625, f. 322v.
} 
le había dedicado gran parte de su trabajo desde su llegada al noroeste novohispano. Pérez de Ribas destaca que la preparación de su muerte le llevó siete años y que debido a la gota le era cada vez más difícil desplazarse. A pesar de ello estuvo en la misión la última cuaresma de su vida en 1634, habiendo fallecido en el Colegio de Sinaloa. ${ }^{49}$

\section{Conclusiones}

Es importante destacar el rol que ejerció Hernando de Villafañe durante medio siglo al servicio de la provincia Mexicana de la Compañía de Jesús, desde su llegada en 1584, hasta su muerte medio siglo después, y cuya figura aparece desdibujada a pesar de ser uno de los protagonistas del trabajo de la orden entre los indios, cubriendo gran parte de los generalatos de Claudio Acquaviva y Mutio Vitelleschi.

¿Cuáles son los aspectos que hicieron de su ejercicio un referente en los orígenes de la Compañía de Jesús en el Noroeste novohispano? Sin duda uno de ellos es que su espíritu misionero reflejaba el impulso que en esa época estimulaba el propio general de la orden, Claudio Aquaviva, en Roma. Es muy significativo el hecho de que su incorporación al trabajo misionero haya venido de origen, al unirse en España al padre Francisco Vaez, que había ido de procurador de la Provincia Mexicana a Roma y es que, vale decir, no todos los jesuitas que llegaron a Nueva España buscaban la misión y no pocos preferían la comodidad de los colegios y el mero trabajo intelectual. De esta forma fue perfilando el perfil de misionero en cuanto llegó a México mediante su estancia en Pátzcuaro y la experiencia viva del trabajo con los indios en compañía de otro de los primeros grandes misioneros de la Provincia Mexicana como fue el padre Juan Ferro.

Uno de los elementos que le caracterizó fue el hecho de ser un padre lengua, lo cual tendió un puente de entendimiento con los indios tanto en Pátzcuaro como en Sinaloa, de cuya relación siempre se dijo fue buena, logrando el jesuita el respeto y la confianza de éstos, que le acompañaron en grandes empresas como lo fue la edificación de los templos misionales pero también en la mantención de relaciones de paz con los españoles a partir de sus primeras misiones. Villafañe ejerció en la época del gran impulso misionero de la orden y su liderazgo no solo permitió la consolidación de los pueblos de misión, sino del propio colegio de Sinaloa del que fue primer rector en 1616 y cuya titularidad le fue asignada en diversos momentos a pesar de su empeño y reiteradas peticiones por que no se le encargara del "mando de otros".

Al desarrollar el mismo el trabajo misional, permaneciendo al lado de los indios de forma regular, a la vez que desempeñaba funciones de superior en distintas escalas de la administración jesuítica se convirtió en referente para el resto de los jesuitas tocante a la construcción de los templos misionales, el aprendizaje de la lengua nativa y la forma de tratar a los indios logrando su amistad y cooperación tocante a las políticas de conquista dirigidas por las autoridades civiles hacía territorios más septentrionales como fueron los ríos Zuaque, Mayo y Yaqui en las que la Compañía de Jesús jugó un rol clave.

Cabe aquí preguntarse sobre el papel de estos hombres cuyo trabajo al ras del suelo se ve reflejado en las políticas seguidas por la Compañía de Jesús mediante la comunicación directa con los superiores, tanto en México como en Roma. Su experiencia fue atendida no solo mediante las cartas al general, sino que aquel -ya no Aquaviva

${ }^{49}$ Pérez de Ribas, 1992: 349. 
sino Mutio Vitelleschi en 1620- tuvo oportunidad de escucharlo muy de cerca siendo procurador. El gran ciclo se cierra de alguna forma cuando a su regreso de la ciudad eterna y realizar las gestiones ante el Consejo de Indias logra la aprobación de un contingente de nuevos misioneros que requería la expansión al norte, tanto en el centro de la Nueva Vizcaya como en la lejana Sonora.

\section{Bibliografía}

\section{Fuentes primarias}

"Relación de las misiones que la Compañía tiene en el Reyno y provincia de la Nueva Vizcaya, en la Nueva España, hecha el año de 1678 con ocasión de la visita general de ellas que por orden del padre provincial Thomas Altamirano hizo el padre visitador Juan Ortiz Zapata, de la misma Compañía”, Archivo General de la Nación (México), Misiones 26, fs 241-269v.

Archivum Romanum Societatis Iesu, México 02, Epistolario del General, 1599-1638.

\section{Fuentes secundarias}

Alegre, Francisco Javier (1956-1958). Historia de la provincia de la Compañía de Jesús de Nueva España, Tomo I (1566-1596) y II (1597-1639). Roma: Institutum Historicum Societatis Iesu.

Bolton, Herbert Eugene (2001) [1936]. Los confines de la cristiandad. Una biografía de Eusebio Francisco Kino, S.J., misionero y explorador de Baja California y la Pimería Alta. México: Universidad de Sonora, Universidad Autónoma de Baja California, Universidad de Colima, Universidad de Guadalajara, Colegio de Sinaloa, Editorial México Desconocido.

Carpenter, John P. (1999). "Las culturas indígenas de Sinaloa en el momento del contacto", en José Luis Moctezuma y Elisa Villalpando (coords.), Noroeste de México, antropología de la identidad e historia en el norte de México. México: Instituto Nacional de Antropología e Historia, pp. 119-128.

Castro Osuna, Carlos y Mario M. Cuevas Aramburo (1992). Esoterismo y entretenimiento en Culiacán, primera mitad del siglo XVII. México: El Colegio de Sinaloa.

Cervantes Ahumada, Raúl (1942). Hernando de Villafañe, fundador de Guasave, México: Ayuntamiento de Guasave, Cámara de Comercio de Guasave y Asociación de Agricultores de Guasave.

Decorme Gerard (1941). La obra de los jesuitas mexicanos durante la época colonial (1572-1767), Compendio Histórico, Tomo II, Las Misiones. México: Antigua Librería de José Porrúa e hijos.

Félix Zubillaga (ed.) (1959-1973). Monumenta Mexicana II, III, IV y V, Roma: Institutum Historicum Societatis Iesu.

García de la Foz, José (2002) [1867]. Crónica general de España, o sea historia ilustrada y descriptiva de sus provincias, sus poblaciones más importantes de la 
península y de ultramar, Crónica de la provincia de León. Valladolid: Editorial Maxtor.

Gómez Padilla, Gabriel (2006). "Las misiones del noroeste. Otra visión de la educación jesuítica", en Revista Latinoamericana de Estudios Educativos. México, Vol. XXXVI, No. $1-2,1^{\circ}$ y $2^{\circ}$ semestre, pp. 49-73.

Gutiérrez Casillas, José (1600-1699). Diccionario bio-bibliográfico de la Compañía de Jesús en México. México: Editorial Tradición.

López Castillo, Gilberto (2013). "De la ciudad eterna a las misiones más remotas: comunicación epistolar de los generales con los jesuitas de Sinaloa, 1591-1630", en IHS. Antiguos Jesuitas en Iberoamérica, Córdoba, Argentina, Vol. 1, No. 2, pp. 121-136.

(2013). "Los mecanismos institucionales de la administración jesuítica en Nueva Vizcaya y la superintendencia de misiones de la Tierra Adentro", en Letras históricas, Número 8, primavera-verano, pp. 15-39.

Lovay, Silvana M. y Carlos A. Page (2013). "El regreso del padre Diego León de Villafañe, último jesuita de la provincia del Paraguay", en IHS. Antiguos Jesuitas en Iberoamérica, Córdoba, UNC-CONICET, Vol. 1, No. 2, pp. 155-169.

Nakayama, Antonio (ed.) (1992). La conquista de Sinaloa. La relación de Antonio Ruiz, Culiacán. México: Colegio de Bachilleres del Estado de Sinaloa, Centro de Estudios Históricos del Noroeste.

Ortega Noriega, Sergio (2003). Un ensayo de historia regional, el noroeste de México, 1531-1880. México: UNAM.

Pérez de Ribas, Andrés (1992). Historia de los triumphos de nuestra santa fee (edición facsimilar), estudio introductorio, apéndices y notas de Ignacio Guzmán Betancourt. México: Siglo XXI Editores-DIFOCUR.

Rodríguez, Miguel Ángel (1991). Monumenta Mexicana VIII. Roma: Institutum Historicum Societatis Iesu.

Rodríguez Villarreal, Juan José (2013). "La superioridad jesuítica frente a sus misiones de la provincia de Sinaloa (1591-1753), en José Carlos Zazueta Manjarrez (Coord.), Seminario la religión y los jesuitas en el Noroeste novohispano, Culiacán: El Colegio de Sinaloa, pp. 13-41.

Santos Ramírez, Víctor Joel (2015). Las misiones jesuíticas de Sinaloa. Pasado y presente de los monumentos históricos. México: Instituto Nacional de Antropología e Historia, Centro INAH-Sinaloa. 